\title{
Exacerbation of Dermatomyositis with Recurrence of Rectal Cancer: A Case Report
}

\author{
Yuka Nagano Yasuhiro Inoue Tadanobu Shimura Hiroyuki Fujikawa \\ Yoshinaga Okugawa Junichiro Hiro Yuji Toiyama Koji Tanaka \\ Yasuhiko Mohri Masato Kusunoki \\ Division of Reparative Medicine, Department of Gastrointestinal and Pediatric Surgery, \\ Institute of Life Sciences, Mie University Graduate School of Medicine, Tsu, Japan
}

\section{Key Words}

Dermatomyositis · Dysphagia · Rectal cancer · Chemotherapy

\begin{abstract}
Dermatomyositis (DM) is a rare idiopathic inflammatory myopathy characterized by cutaneous and muscle manifestations. The association between DM and malignancy has been well recognized for many years. The clinical course of paraneoplastic DM may be affected by malignancies, although the cause and effect relationship between exacerbation of DM and cancer progression is uncertain. Herein, we report a 44-year-old woman who presented with progressive DM associated with rectal cancer. After curative resection of rectal cancer, DM symptoms resolved. Three months after surgery, blood test surveillance showed elevation of serum carcinoembryonic antigen levels, although the patient remained asymptomatic. One month later she had a DM flare-up, and multiple lung and liver metastases were found. She immediately underwent cancer chemotherapy with prednisolone therapy for DM. However, her condition deteriorated and she was unable to swallow. Percutaneous endoscopic gastrostomy was constructed, allowing alimentation and oral delivery, which made it possible to keep her on chemotherapy. She had remarkable response for unresectable metastases 8 weeks after the administration of chemotherapy. Seven months after onset of recurrence, her condition improved considerably and she had stable disease. Moreover, she can now eat food of soft consistency. Our case provides further support for the clinical importance of cancer chemotherapy for patients who have progressive DM and unresectable rectal cancer.
\end{abstract}


Nagano et al.: Exacerbation of Dermatomyositis with Recurrence of Rectal Cancer: A Case Report

\section{Introduction}

Dermatomyositis (DM) is a rare idiopathic inflammatory myopathy characterized by cutaneous and muscle manifestations. The association between DM and malignancy has been well recognized for many years. Approximately $70 \%$ of all cases of cancer associated with DM and polymyositis are adenocarcinoma, including colorectal cancer (CRC) [1]. One type of DM is thought to be paraneoplastic syndrome and the clinical course of DM may follow that of malignancy. Several reports revealed that malignancy resection improved paraneoplastic DM syndromes [2, 3]. However, when patients have unresectable metastases, DM flare-ups may make the patient unable to tolerate modern effective chemotherapy, which results in poor prognosis.

We herein present a report of successful treatment of rapid, progressive liver and lung metastases from rectal cancer and coexistence of DM exacerbation with dysphagia by strict treatment with both bevacizumab-containing chemotherapy and steroid therapy with construction of a gastrostomy.

\section{Case Report}

A 44-year-old woman presented with progressive skin rash on her face and muscle pain in all four limbs over a 1-month period. Within 1 month, the weakness appeared and progressed, which made it difficult to walk and caused joint pain. A clinical diagnosis of DM was made, which was supported by raised muscle-associated enzyme levels, and continuous oral administration of prednisolone $20 \mathrm{mg}$ /day was begun. There was initial improvement in the skin lesions and the elevated pre-admission serum levels of muscle-associated enzymes, including aspartate transaminase, creatine phosphokinase, lactate dehydrogenase, myoglobin and aldolase, decreased, but the muscle weakness persisted, albeit with some improvement. On further examination, colonoscopy study and histopathological examination revealed the presence of adenocarcinoma of the rectum; therefore, the patient was given a diagnosis of colon cancer-associated DM. She was treated with radical low anterior resection, and the final diagnosis was moderate differentiated adenocarcinoma, pT3N0M0, stage II rectal cancer. Following excision of the rectal cancer, the characteristic features of the skin rash and the muscle-associated enzyme levels improved and the patient was discharged home after a few days. As her weakness and rash resolved and creatine phosphokinase levels normalized, tapering down of steroids became possible.

Three months after surgery, blood tests for surveillance showed elevation of serum carcinoembryonic antigen (CEA) levels, although the patient remained asymptomatic. There was a 1-month lag between the initial elevation of CEA and DM flare-up, including muscle pain, limb joint pain and facial erythema. She was readmitted to our hospital, and $30 \mathrm{mg} /$ day of prednisolone was administered. Serum muscle-associated enzyme levels also increased gradually, as did serum CEA (fig. 1). A computed tomography (CT) scan also showed multiple lung and liver metastases. We suspected paraneoplastic manifestation recurrences where the rectal cancer had relapsed. She was immediately administered concomitant systemic cancer chemotherapy with prednisolone therapy for DM. A combination regimen of modified FOLFOX6 plus bevacizumab was initially commenced, but the patient's condition deteriorated. She became very weak, needed help in all activities of daily living, was unable to swallow and started drooling. She received $500 \mathrm{mg}$ prednisolone i.v. daily for 3 days for the worsening DM and cancer chemotherapy was continued. As there was no improvement in swallowing, a percutaneous endoscopic gastrostomy was also constructed, allowing alimentation 
Nagano et al.: Exacerbation of Dermatomyositis with Recurrence of Rectal Cancer: A Case Report

and oral delivery, which made it possible to maintain her chemotherapy. In addition, oxaliplatin was removed after the first cycle of modified FOLFOX6 due to grade 3 thrombocytopenia, but the leucovorin/5-FU/bevacizumab regimen was continued. A CT scan after two chemotherapy cycles showed remarkable response, and CEA fell from 65.1 to $5.2 \mathrm{ng} / \mathrm{ml}$ (fig. 1). The skin lesions and muscle weakness gradually improved, serum levels of muscleassociated enzymes also normalized, and early tumor shrinkage of the metastatic sites was noted. The patient was discharged from the hospital after a fourth cycle of chemotherapy, and the chemotherapy was continued in an outpatient clinic. Seven months after recurrence onset, the patient's condition had improved considerably (stable disease of cancer recurrence), and she can now eat food with soft consistency.

\section{Discussion}

The clinical course of paraneoplastic DM may be affected by malignancy, although the cause-effect relationship between DM exacerbation and cancer progression is uncertain. The malignant process is usually diagnosed within a year from onset of myopathy, whose course in turn parallels that of the tumor [2]. Successful treatment of such malignancies can lead to resolution of paraneoplastic symptoms. Several reports have shown that resection of the tumor can lead to resolution of paraneoplastic DM symptoms [4,5]. Our patient's DM symptoms also resolved after the initial surgical treatment of rectal cancer. We also found the liver and lung metastases from rectal cancer by using blood examination of CEA followed by CT when a DM flare-up appeared approximately 4 months after surgery. This is consistent with published reports that recurrent DM symptoms following curative cancer therapy require reinvestigation, even in the absence of symptoms of malignancies [2,6]. However, when the patient has an unresectable malignancy, cancer chemotherapy administration difficulties exist because of poor performance status from paraneoplastic DM and occasional steroid refractoriness of DM symptoms [7]. Furthermore, paraneoplastic DM triggered and exacerbated by anti-cancer drugs, such as 5-FU, has also been reported [8].

In our case, the patient had multiple metastases with rapid progression that occurred with the DM flare-up and resulted in dysphagia. It seems likely that coexistence with dysphagia and cancer progression is not uncommon, because it is estimated that $10-73 \%$ of patients with inflammatory myopathies suffer from dysphagia [9], and a recent metaanalysis showed that characteristics including dysphagia may influence cancer development in patients with DM [10]. Despite the notion that severe dysphagia indicates poor prognosis in patients with DM, the condition can be resolved if treated early using high-dose corticosteroid or insertion of percutaneous gastrostomy $[9,11]$. However, when patients have unresectable malignancies, it is very hard to treat the DM exacerbation and the malignancy at the same time. Although some reports succeeded in handling both cancer chemotherapy and medication for paraneoplastic DM flare-up, including ovarian and breast cancer [12, 13], to our knowledge, this is the first report of DM with CRC. Herein, we describe a case of exacerbated DM responsive to combination therapy with corticosteroids, insertion of percutaneous gastrostomy and bevacizumab-containing chemotherapy in a patient with DM and recurrent rectal cancer. Interestingly, serum CEA was initially abnormal prior to the elevation of the muscle-associated enzymes, and tumor marker velocity was higher than that of muscleassociated enzymes (fig. 1), although the progressive DM and recurrent malignancy seemingly occurred at the same time. Therefore, we thought that the main cause of DM manifestations was tumor recurrence and made immediate cancer chemotherapy a higher priority than DM therapy. We used FOLFOX6 plus bevacizumab for the recurrent rectal cancer be- 
Nagano et al.: Exacerbation of Dermatomyositis with Recurrence of Rectal Cancer: A Case Report

cause the patient was diagnosed with KRAS mutant type adenocarcinoma. Even after deterioration of her condition, we continued infusional 5-FU plus bevacizumab because National Comprehensive Cancer Network (NCCN) guidelines recommend the use of this regimen in CRC patients when intensive therapy is not appropriate [14]. Systemic chemotherapy for this disease under strict DM management was found to be tolerable, with encouraging response rates.

In conclusion, we herein report the first documented case of exacerbated DM occurring in association with unresectable recurrence from rectal cancer. Our case also provides further support for the clinical importance of cancer chemotherapy for patients who have progressive DM and unresectable CRC. Of course, we are unable to make generalizations regarding this therapy based on this single case. However, accumulation of case data may help to establish a strategy for metastatic CRC patients with neoplastic DM to prolong survival.

\section{Statement of Ethics}

Written informed consent was obtained from the patient for publication of this case report.

\section{Disclosure Statement}

The authors have no conflicts of interest to declare.

\section{References}

1 Bohan A, Peter JB: Polymyositis and dermatomyositis (first of two parts). N Engl J Med 1975;292:344-347.

$\checkmark 2$ Samuels N, Applbaum YH, Esayag Y: Paraneoplastic necrotizing myopathy and dermatomyositis in a patient with rectosigmoid carcinoma. Rheumatol Int 2013;33:1619-1621.

3 Osako T, Ito Y, Morimatsu A, Tada K, Sakurai N, Takahashi S, Akiyama F, Iwase T, Hatake K: Flare-up of dermatomyositis along with recurrence of breast cancer. Breast J 2007;13:200-202.

4 Hatada T, Aoki I, Ikeda H, Tamura T, Okada K, Nakai T, Utsunomiya J: Dermatomyositis and malignancy: case report and review of the Japanese literature. Tumori 1996;82:273-275.

5 Rosa F, Ferrari M, Buschiazzo A, Lanfranco E: Myopathy as paraneoplastic syndrome of colon malignancy in an elderly patient. Aging Clin Exp Res 2013;25:221-223.

6 Fitzpatrick J, Wallace WA, Lang S, Aboumarzouk OM, Windsor P, Nabi G: Recurrent dermatomyositis manifesting as a sign of recurrent transitional cell carcinoma of urinary bladder: long-term survival. Urol Ann 2014;6:264-266.

7 Goyal S, Nousari HC: Paraneoplastic amyopathic dermatomyositis associated with breast cancer recurrence. J Am Acad Dermatol 1996;41:874-875.

-8 Kawachi Y, Koike Y, Kano T, Furuta J, Fujisawa Y, Nakamura Y, Ishii Y, Takahashi T, Otsuka F: Paraneoplastic dermatomyositis triggered and exacerbated by oral 5-fluorouracil administration. Eur J Dermatol 2008;18:195-196.

-9 Dagan A, Markovits D, Braun-Moscovici Y, Rozin A, Toledano K, Balbir-Gurman A: Life-threatening oropharyngeal aphagia as the major manifestation of dermatomyositis. Isr Med Assoc J 2013;15:453-455.

10 Wang J, Guo G, Chen G, Wu B, Lu L, Bao L: Meta-analysis of the association of dermatomyositis and polymyositis with cancer. Br J Dermatol 2013;169:838-847.

11 Ocampo C, Segwagwe ML, Deonarain J, Cainelli F, Vento S: A case of severe dermatomyositis in an African woman. Isr Med Assoc J 2014;16:329-330.

12 Girouard SD, Velez NF, Penson RT, Massarotti EM, Vleugels RA: Panniculitis associated with dermatomyositis and recurrent ovarian cancer. Arch Dermatol 2012;148:740-744.

13 Murgić J, Prpić M, Kirac I, Camino-Varela AM, Bolanca A, Kusić Z: Dermatomyositis as paraneoplastic syndrome of peritoneal and ovarian relapse after long-term complete remission in patient with metastatic bilateral breast cancer. Coll Antropol 2012;36:325-329. 
Nagano et al.: Exacerbation of Dermatomyositis with Recurrence of Rectal Cancer: A Case Report

14 Engstrom PF; National Comprehensive Cancer Network: Systemic therapy for advanced or metastatic colorectal cancer: National Comprehensive Cancer Network guidelines for combining anti-vascular endothelial growth factor and anti-epidermal growth factor receptor monoclonal antibodies with chemotherapy. Pharmacotherapy 2008;28(11 Pt 2):18S-22S.

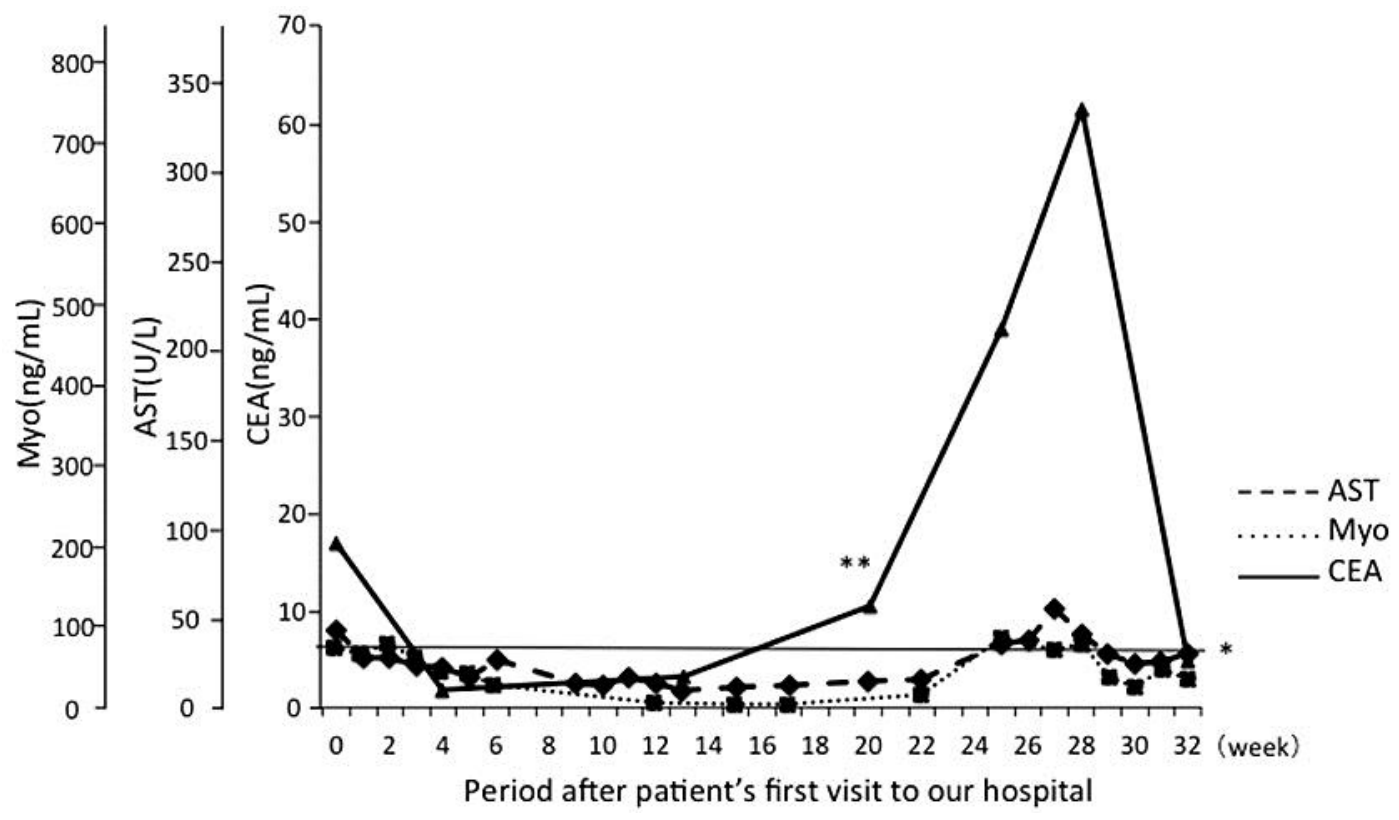

Fig. 1. Serum muscle-associated enzyme level and serum CEA level during treatment. * The line indicates the upper value of normal range. ${ }^{* *}$ Serum CEA was initially raised, but the patient remained asymptomatic. Serum muscle-associated enzyme levels increased gradually 1 month after elevation of serum CEA. However, CEA fell to within the normal range after two cycles of chemotherapy. AST = Aspartate transaminase; Myo = myoglobin. 